NASA/CR-2004-212892

\title{
Computation of Low Speed Cavity Noise
}

Ching Y. Loh

Taitech, Inc., Beavercreek, Ohio 
Since its founding, NASA has been dedicated to the advancement of aeronautics and space science. The NASA Scientific and Technical Information (STI) Program Office plays a key part in helping NASA maintain this important role.

The NASA STI Program Office is operated by Langley Research Center, the Lead Center for NASA's scientific and technical information. The NASA STI Program Office provides access to the NASA STI Database, the largest collection of aeronautical and space science STI in the world. The Program Office is also NASA's institutional mechanism for disseminating the results of its research and development activities. These results are published by NASA in the NASA STI Report Series, which includes the following report types:

- $\quad$ TECHNICAL PUBLICATION. Reports of completed research or a major significant phase of research that present the results of NASA programs and include extensive data or theoretical analysis. Includes compilations of significant scientific and technical data and information deemed to be of continuing reference value. NASA's counterpart of peerreviewed formal professional papers but has less stringent limitations on manuscript length and extent of graphic presentations.

- TECHNICAL MEMORANDUM. Scientific and technical findings that are preliminary or of specialized interest, e.g., quick release reports, working papers, and bibliographies that contain minimal annotation. Does not contain extensive analysis.

- CONTRACTOR REPORT. Scientific and technical findings by NASA-sponsored contractors and grantees.
- CONFERENCE PUBLICATION. Collected papers from scientific and technical conferences, symposia, seminars, or other meetings sponsored or cosponsored by NASA.

- SPECIAL PUBLICATION. Scientific, technical, or historical information from NASA programs, projects, and missions, often concerned with subjects having substantial public interest.

- TECHNICAL TRANSLATION. Englishlanguage translations of foreign scientific and technical material pertinent to NASA's mission.

Specialized services that complement the STI Program Office's diverse offerings include creating custom thesauri, building customized databases, organizing and publishing research results ... even providing videos.

For more information about the NASA STI Program Office, see the following:

- Access the NASA STI Program Home Page at http://www.sti.nasa.gov

- E-mail your question via the Internet to help@sti.nasa.gov

- Fax your question to the NASA Access Help Desk at 301-621-0134

- Telephone the NASA Access Help Desk at 301-621-0390

- Write to:

NASA Access Help Desk

NASA Center for AeroSpace Information 7121 Standard Drive

Hanover, MD 21076 
NASA/CR-2004-212892

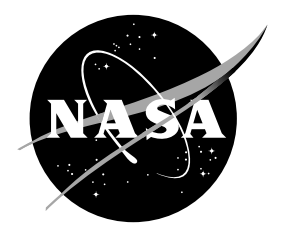

\section{Computation of Low Speed Cavity Noise}

Ching Y. Loh

Taitech, Inc., Beavercreek, Ohio

Prepared for the

42nd Aerospace Sciences Meeting and Exhibit

sponsored by the American Institute of Aeronautics and Astronautics

Reno, Nevada, January 5-8, 2004

Prepared under Contract NAS3-03072

National Aeronautics and

Space Administration

Glenn Research Center 


\section{Acknowledgments}

This work received support from the Supersonic Propulsion Technology Project Office of NASA Glenn Research Center.

This report is a formal draft or working paper, intended to solicit comments and ideas from a technical peer group.

\section{This work was sponsored by the Low Emissions Alternative}

Power Project of the Vehicle Systems Program at the NASA Glenn Research Center.

Available from

NASA Center for Aerospace Information 7121 Standard Drive

Hanover, MD 21076
National Technical Information Service 5285 Port Royal Road Springfield, VA 22100 


\title{
COMPUTATION OF LOW SPEED CAVITY NOISE
}

\author{
Ching Y. Loh* \\ Taitech, Inc. \\ Beavercreek, Ohio 45430
}

\begin{abstract}
Cavity noise is an important noise source. It is generated primarily by wall-jet shear layer and vortex shedding and an aeroacoustic feedback loop. At low flow speed, the situation is further complicated by the presence of the Helmholtz resonance of the cavity.

In this paper, the space-time conservation element and solution element (CE/SE) method is employed to numerically study the near-field noise of a cavity at low flow speeds. The numerical approach is of the MILES type (monotonically integrated large eddy simulation) [4$6]$. The computed frequencies and sound pressure levels (SPL) compare well with the experimental findings.
\end{abstract}

\section{Introduction}

Noise prediction and noise control are important for aerospace and automobile industries. The noises due to aeronautics at high speed, such as airfoil slat, landing gear and openings, etc. are some of the major noise sources in aeroacoustics. For ground transportation at low Mach number, the automobile industry is concerned with the noises produced by the door gaps, the side mirror and the aerial and how they affect the comfort in the car. All these noises may be categorized as airframe noises that are generated by the interaction between the vortex streets in the turbulent wake or between the vortices and the solid body edge(s). The phenomena are further complicated by a possible aeroacoustic feedback loop or a possible Helmholtz fluid resonance.

Cavity noise is one of the most important airframe noises. When flow passes over a cavity or opening, due to vortex shedding at the upstream edge of the cavity, intensive tone noises may be generated. Strong tonal oscillations occur in a feedback loop between the two edges of the cavity opening. First, the vortices generated and

*Taitech Inc., Member AIAA shed from the upstream edge of the cavity convect downstream, impinge on the other edge, and produce acoustic waves. Then, as the acoustic waves propagate either inside or outside of the cavity to the upstream edge, where receptivity of the wall jet shear layer is high, a new instability wave (vortex) is stimulated and shed, a feedback cycle is thus completed. The tone noise generated in this way may be categorized as fluid-dynamic oscillation noise. Most high speed flow (supersonic, transonic, or high subsonic flows) noises are generated by the fluid dynamic feedback oscillation mode. However, at low flow speed [1 - 3], depending on the geometry of the cavity, another type of tone noise due to fluid resonant oscillation within the cavity may occur. The sound waves inside the cavity may be longitudinal or transverse depending on the aspect ratio of the cavity. For a deep cavity at low flow speed, both major tone noise generating mechanisms may coexist.

Numerical investigations of cavity noise based on direct numerical simulation (DNS) need very fine grids and are hence expensive. Large eddy simulations (LES) allow a coarser grid since only large eddies are resolved and the smaller ones are modeled by a subgrid scale (SGS) model.

In the present paper, a MILES (monotonically integrated large eddy simulation, [ 4 - 6]) type approach is used to investigate a typical low speed cavity noise problem. In all the cases, the grid may not be fine enough for the Kolmogorov scale of turbulence but is fine enough for the large turbulence structures (large eddies or instability waves ) associated with the tone noise frequencies. The MILES approach appears somewhat similar to LES, but there is no explicit filtering since the cell-averaging process is already equivalent to some spatial filtering. Due to certain built-in numerical dissipation in a MILES type finite volume scheme, the SGS (subgrid scale) model may not be needed. The recent space-time conservation element and solution element method (CE/SE) [7$8]$ is a MILES type finite volume method with generally less numerical dissipation and is adopted for the com- 
putation. As demonstrated in the previous papers, the $\mathrm{CE} / \mathrm{SE}$ scheme is well suited for aeroacoustics computation $[9,13]$. Because of the CE/SE non-reflecting boundary conditions (NRBC), which are based on the first principle of plane wave propagation [14], a smaller near field computational domain can be used in the present numerical simulation and helps to save both memory and CPU time.

The 2-D unstructured-grid Navier-Stokes (N-S) $\mathrm{CE} / \mathrm{SE}$ scheme used here is briefly discussed in Section 2. Section 3 illustrates the noise problem of a rectangular cavity with an overhang at low flow speed, including the initial and boundary conditions. The numerical results are presented and compared to available experimental findings [15] in Section 4. They are then compared to the experiment data. Concluding remarks are drawn in Section 5.

\section{The Numerical Scheme}

In the present 2-D MILES approach, the spacetime conservation element and solution element, CE/SE method is chosen. The CE/SE scheme is a low order (second order accurate in time and space) scheme with low dissipation. A detailed description of the method can be found in [7- 8]. In this Section, a brief sketch of the scheme is given.

\subsection{Conservation form of the 2-D unsteady compressible Navier-Stokes equations}

In general, the CE/SE method systematically solves a set of integral equations derived directly from the physical conservation laws and naturally captures shocks and other discontinuities in the flow. Both conservative variables and their derivatives are computed simultaneously as unknowns.

Consider a dimensionless conservation form of the unsteady 2-D Navier-Stokes equations of a perfect gas. Let $\rho, u, v, p$, and $\gamma$ be the density, streamwise velocity component, transverse velocity component, static pressure, and constant specific heat ratio, respectively. The 2-D Navier-Stokes equations then can be written in the following vector form:

$$
\boldsymbol{U}_{t}+\boldsymbol{F}_{x}+\boldsymbol{G}_{y}=\mathbf{0},
$$

where $x, y$, and $t$ are the streamwise and transverse coordinates and time, respectively. The conservative flow variable vector $\boldsymbol{U}$ and the flux vectors in the streamwise and the normal ( $y$ ) directions, $\boldsymbol{F}$ and $\boldsymbol{G}$, are given by:

$$
\boldsymbol{U}=\left(\begin{array}{c}
U_{1} \\
U_{2} \\
U_{3} \\
U_{4}
\end{array}\right), \quad \boldsymbol{F}=\left(\begin{array}{c}
F_{1} \\
F_{2} \\
F_{3} \\
F_{4}
\end{array}\right), \quad \boldsymbol{G}=\left(\begin{array}{c}
G_{1} \\
G_{2} \\
G_{3} \\
G_{4}
\end{array}\right),
$$

with

$$
U_{1}=\rho, \quad U_{2}=\rho u, \quad U_{3}=\rho v,
$$

$$
U_{4}=p /(\gamma-1)+\rho\left(u^{2}+v^{2}\right) / 2 .
$$

The flux vectors are further split into inviscid and viscous fluxes:

$$
\mathbf{F}=\mathbf{F}_{\mathbf{i}}-\mathbf{F}_{\mathbf{v}}, \mathbf{G}=\mathbf{G}_{\mathbf{i}}-\mathbf{G}_{\mathbf{v}},
$$

where the inviscid fluxes are the same as in the Euler equations:

$$
\begin{gathered}
F_{i 1}=U_{2}, \\
F_{i 2}=(\gamma-1) U_{4}+\left[(3-\gamma) U_{2}^{2}-(\gamma-1) U_{3}^{2}\right] / 2 U_{1}, \\
F_{i 3}=U_{2} U_{3} / U_{1}, \\
F_{i 4}=\gamma U_{2} U_{4} / U_{1}-(\gamma-1) U_{2}\left[U_{2}^{2}+U_{3}^{2}\right] / 2 U_{1}^{2}, \\
G_{i 1}=U_{3}, \quad G_{i 2}=U_{2} U_{3} / U_{1}, \\
G_{i 3}=(\gamma-1) U_{4}+\left[(3-\gamma) U_{3}^{2}-(\gamma-1) U_{2}^{2}\right] / 2 U_{1}, \\
G_{i 4}=\gamma U_{3} U_{4} / U_{1}-(\gamma-1) U_{3}\left[U_{2}^{2}+U_{3}^{2}\right] / 2 U_{1}^{2},
\end{gathered}
$$

and the viscous fluxes are:

$$
\begin{gathered}
F_{v 1}=0, \quad F_{v 2}=\mu\left(2 u_{x}-\frac{2}{3} \nabla \cdot \mathbf{V}\right), \\
F_{v 3}=\mu\left(v_{x}+u_{y}\right), \\
F_{v 4}=\mu\left[2 u u_{x}+\left(u_{y}+v_{x}\right) v-\frac{2}{3}(\nabla \cdot \mathbf{V}) u+\right. \\
\left.\frac{\gamma}{\operatorname{Pr}} \frac{\partial}{\partial y}\left(\frac{U_{4}}{U_{1}}-\frac{u^{2}+v^{2}}{2}\right)\right], \\
G_{v 1}=0, \quad G_{v 2}=\mu\left(v_{x}+u_{y}\right), \\
G_{v 3}=\mu\left(2 v_{y}-\frac{2}{3} \nabla \cdot \mathbf{V}\right), \\
G_{v 4}=\mu\left[2 v v_{y}+\left(u_{y}+v_{x}\right) u-\frac{2}{3}(\nabla \cdot \mathbf{V}) v+\right. \\
\left.\frac{\gamma}{\operatorname{Pr}} \frac{\partial}{\partial y}\left(\frac{U_{4}}{U_{1}}-\frac{u^{2}+v^{2}}{2}\right)\right],
\end{gathered}
$$

where $u, v, u_{x}, u_{y}, v_{x}, v_{y}$ are respectively the $x-$ and $y-$ flow velocity components and their derivatives, which can be written in terms of the conservative variables $U_{1}, U_{2}, U_{3}$ and $U_{4}$, with $\operatorname{Pr}(=0.72)$ being the Prandtl number, $\mu$ the viscosity, the velocity divergence

$$
\nabla \cdot \mathbf{V}=u_{x}+v_{y} .
$$

By considering $(x, y, t)$ as coordinates of a threedimensional Euclidean space, $E_{3}$, and using the Gauss divergence theorem, it follows that Eq. (1) is equivalent to the following integral conservation law:

$$
\oint_{S(V)} \boldsymbol{H}_{m} \cdot \mathrm{d} \mathbf{S}=\mathbf{0}, \quad \mathbf{m}=\mathbf{1}, \mathbf{2}, \mathbf{3}, \mathbf{4},
$$

where $S(V)$ denotes the surface around a volume $V$ in $E_{3}$ and $\boldsymbol{H}_{m}=\left(F_{m}, G_{m}, U_{m}\right)$. 


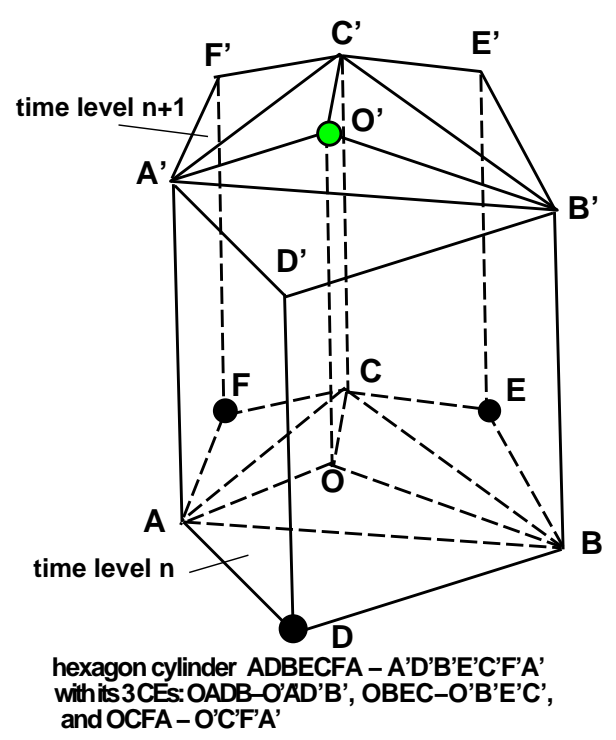

Figure 1: $\mathrm{CE} / \mathrm{SE}$ unstructured grid in space-time $E_{3}$ space.

\subsection{Updating with a compact cell stencil}

The CE/SE scheme is constructed to take advantage of an unstructured triangle grid. The unstructured geometry used with the CE/SE scheme is illustrated in Fig. 1. Here, $\triangle A B C$ is a typical triangular cell centered at $O$ (or $O^{\prime}$ at the new time level) and $D, E, F$ are the triangle centers of the neighboring cells. The flow variables at the previous time step are stored at the triangle cell centers. Centers $D, E, F$ form a compact stencil to support the marching of $\mathbf{U}$ at $O$ to the next time level $O^{\prime}$. Three quadrilateral cylinders (conservation elements) are formed by the edges that connect the vertices and the center of the triangle and its three neighbors. In the space-time $E_{3}$ space, Eq. (2) is applied to the hexagon cylinder $A D B E C F-A^{\prime} D^{\prime} B^{\prime} E^{\prime} C^{\prime} F^{\prime}$ of volume $V$ that consists of these 3 quadrilateral cylinder $C E$ s, see Fig 1.

In the CE/SE scheme, the above flux conservation relation, Eq. (2), in space-time is the only mechanism that transfers information between node points. A conservation element $C E$ (here, quadrilateral cylinders) is the finite volume to which Eq. (2) is applied. Discontinuities are allowed to occur in a conservation element. A solution element $S E$ associated with a grid node (e.g., $D$, $E$, or $F$ in Fig. 1) is here a set of interface planes in $E_{3}$ that passes through this node (e.g. $D A A^{\prime} D^{\prime}, D B B^{\prime} D^{\prime}$, $E B B^{\prime} E^{\prime}, E C C^{\prime} E^{\prime}$, etc.). Each surface $S(C E)$ is made up of segments belonging to two neighboring $C E$ 's. Within a given solution element $S E(j, n)$, where $j, n$ are the node index and time step, respectively, the flow variables are not only considered continuous but are also approximated by the linear Taylor series expansions. The surface flux can then be calculated accurately and easily by first evaluating the flux vectors at the geometrical center of the surface through such Taylor expansions.

At time level $n$, the solution variables $\boldsymbol{U}, \boldsymbol{U}_{x}$, and $\boldsymbol{U}_{y}$ are given at the three nodes $D, E, F$ in Fig. 1 and $\boldsymbol{U}, \boldsymbol{U}_{x}$ and $\boldsymbol{U}_{y}$ at $O^{\prime}$ at the new time level $n+1$ are to be computed. In principle, each of the $3 \mathrm{CEs}$ provides 4 scalar equations when Eq. (2) is applied to the element. Hence, the 12 scalar equations needed for the 12 scalar unknowns at $O^{\prime}$ are available. All the unknowns are computed based on these relations. No extrapolations (interpolations) across a stencil of cells are needed.

More details about the unstructured CE/SE method can be found in [8].

\subsection{Non-reflecting boundary condition (NRBC) for CE/SE}

In the CE/SE scheme, NRBCs are constructed based on the first principle of plane wave propagation and the hyperbolicity of the equation system [14]. There are various variants of the NRBCs [9-14], the following are the ones employed in this paper.

For a grid node $(j, n)$ lying at the outer border of the domain, where $j$ is the grid node index number and $n$ the time step number, the Type I NRBC requires that

$$
\left(\boldsymbol{U}_{x}\right)_{j}^{n}=\left(\boldsymbol{U}_{y}\right)_{j}^{n}=0,
$$

while $U_{j}^{n}$ is kept fixed at the initially given steady boundary value. At the outflow boundary, where there are substantial gradients in the $y$ direction, the Type II NRBC requires that

$$
\left(\boldsymbol{U}_{x}\right)_{j}^{n}=0,
$$

while $\boldsymbol{U}_{j}^{n}$ and $\left(\boldsymbol{U}_{y}\right)_{j}^{n}$ are now defined by simple extrapolation from the nearest interior node $j^{\prime}$, i.e.,

$$
\boldsymbol{U}_{j}^{n}=\boldsymbol{U}_{j^{\prime}}^{n} \quad\left(\boldsymbol{U}_{y}\right)_{j}^{n}=\left(\boldsymbol{U}_{y}\right)_{j^{\prime}}^{n}
$$

As will be observed later, these NRBCs are robust enough to allow a near field computation without disturbing or distorting the flow and acoustic fields.

\section{Low-speed Tone Noise of a Cavity with Overhang}

For high speed flows ranging from high subsonic, transonic to supersonic, typical cavity noise examples are the airplane wheel well and weapon bay noises. Therefore, cavity noises are often studied at high flow speed when aeroacoustic feedback loop plays a dominant role. Rossiter [1] has a semi-empirical model to describe the convection of the vortices and hence the feedback tone frequencies for high subsonic flows. However, for ground transport vehicles at low subsonic Mach numbers (typically around or below $\mathrm{M}=0.1$ ), or in the presence of a deep cavity, the noise due to flow-resonant phenomena 


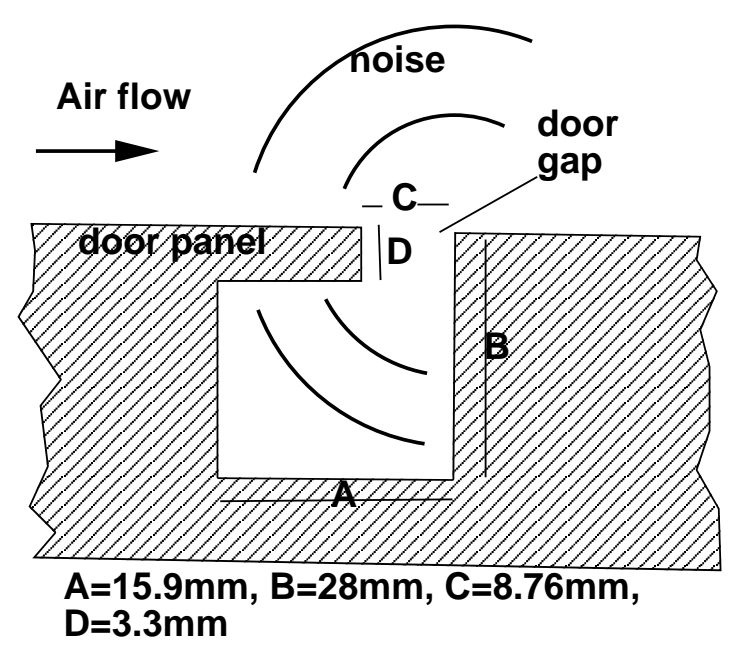

Figure 2: auto-door gap noise problem and the cavity dimensions.

occurs as well. Howe [2] analyzes the cavity noise at low Mach numbers. Chatellier et al. [3] have detailed analysis and experimental data for a rectangular cavity.

In the present paper, for low speed flows with Mach number as low as 0.08 , a cavity with an overhang at the opening (Fig. 2) is investigated. This is a prototype of the automobile door gap noise problem [15].

Figure 2 illustrates the geometric configuration and the actual dimensions of the cavity noise problem. Due to the presence of the overhang tongue, the two oscillation modes, namely, the fluid dynamic oscillation due to aeroacoustic feedback, and the Helmholtz fluid resonance in the cavity may emerge at the same time and compete with each other. The problem is given as a benchmark problem at the 3rd CAA (Computational Aeroacoustics) Workshop [15]. In Fig. 2, C is the length of the cavity opening. $2 \mathrm{C}$ is then chosen as the length scale. The speed of sound $(343 \mathrm{~m} / \mathrm{s})$ and density of the ambient flow are respectively the scales for velocity and density. In the current computation, there are about 85300 triangular cells in the computational domain. The domain ranges $-1 \leqslant x \leqslant 2.1$ and $0 \leqslant y \leqslant 3.2$. The triangles are actually obtained by dividing a rectangular structured mesh cell into 4 pieces. The rectangular cell keeps a uniform size of $\Delta x=0.00625$ and $\Delta y=0.00625$ around the area of the opening and the interior of the cavity (Fig. 3), but grows larger near the outer boundaries. A nondimensional time step size $\Delta t=0.0025$, and a weighted average (van Albada limiter) index $\alpha=0$ are employed.

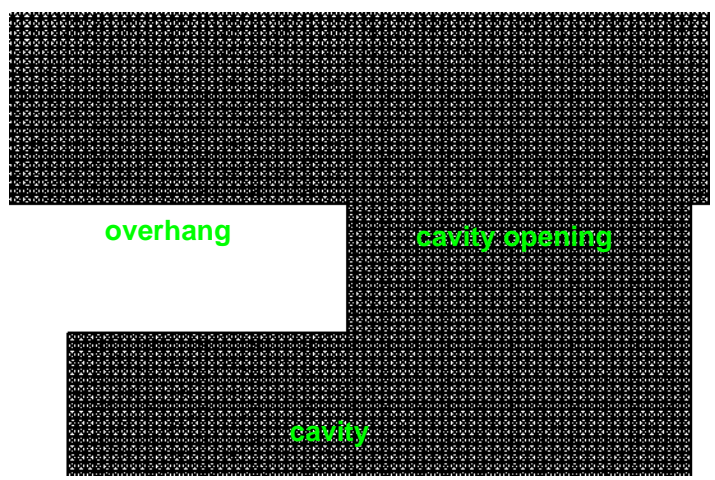

Figure 3: Trangulated grid around the overhang of the cavity.

\subsection{Initial and boundary conditions}

Initially, the flow of the entire domain is set at the ambient conditions, i.e.,

$$
\rho_{0}=1.0, \quad u_{0}=0, \quad v_{0}=0, \quad p_{0}=1 / \gamma,
$$

All spatial derivatives are set to zero.

At the inflow boundary, the flow variables are specified as the uniform 'plug' flow:

$$
\rho_{i}=1.0, \quad u_{i}=M, \quad v_{i}=0, \quad p_{i}=1 / \gamma,
$$

and their spatial derivatives are conveniently set to zero. The Mach numbers range from $M=0.0788$ to $M=$ 0.147 , corresponding respectively to flow speeds of 26.8 $\mathrm{m} / \mathrm{s}$ and $50 \mathrm{~m} / \mathrm{s}$. When a consistency test is conducted in $\S 4.2 .4$, a prescribed profile is imposed on $u$-velocity at the inlet boundary.

The no-slip wall boundary condition is applied to all the solid walls of the cavity body. Boundary condition at the top is the same as the inflow, which also acts as a Type I NRBC. At the outflow boundary, Type II NRBC is imposed.

\section{Numerical Results}

In this section, numerical results for the low speed cavity noises are presented and compared to their respective experimental data. As designed in the experiment, all the data were obtained at a location at the center of the inner left vertical wall of the cavity.

As described earlier, for the overhung cavity considered here, there are two major modes for the tone noises: the fluid dynamic tone noise due to the feedback loop around the cavity opening, and the fluid-resonant tone noises due to the Helmholtz resonance of the cavity. In addition, there could exist other tone noise modes of uncertain origins or broadband noises, making the phenomena rather complicated. For MILES approach, Fureby [6] points out that the numerical result is relatively insensitive to the LES subgrid model but more dependent 


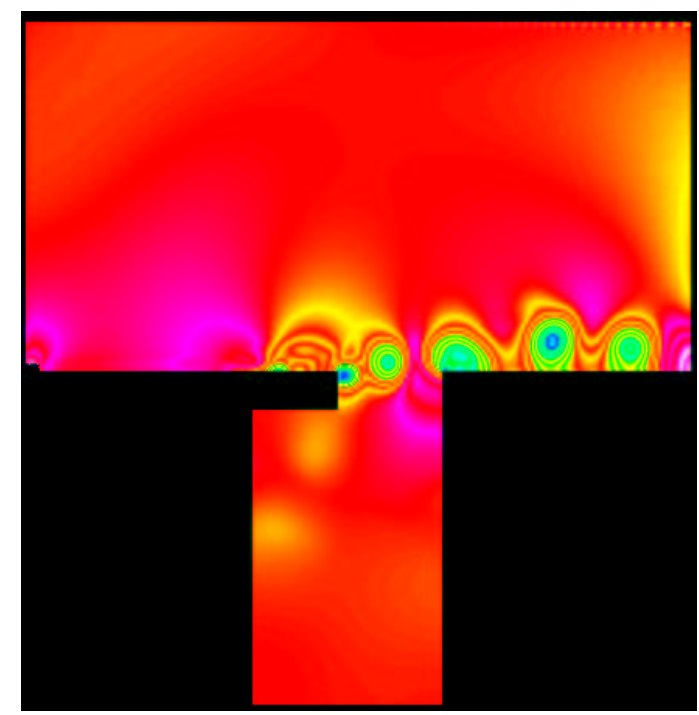

Figure 4: Instantaneous isobars at time step 740,000.

on the grid sizes. Generally, a finer grid yields more details in the acoustic field and higher SPL. It is important to choose a grid fine enough and an appropriate weighted average index number $\alpha$.

In the following, numerical results corresponding to flow speeds of $50 \mathrm{~m} / \mathrm{s}$ and $26.8 \mathrm{~m} / \mathrm{s}$ are presented and compared to the respective experimental data [15]. For the case with flow speed of $50 \mathrm{~m} / \mathrm{s}$, several consistency tests for the numerical work are also conducted. All the SPLs (sound pressure levels) are presented in $\mathrm{dB}$ based on their magnitudes against the standard $20 \mu P a$.

\subsection{Noise at flow speed of $50 \mathrm{~m} / \mathrm{s}$}

In order to have a sizable sampling time series for FFT (fast Fourier transform) analysis, with non-dimensional time step size $\Delta t=0.0025$, over half million time steps are run. Figure 4 - Figure 5 demonstrate the isobars and v- velocity contours at time step 740,000. The unsteadiness and the large scale turbulence or instability waves are clearly displayed. In these figures, it is interesting to observe that the onset of the instability waves (vortices) does not occur right at the lip of the overhung tongue of the cavity, as expected for feedback oscillation, but occurs somewhat upstream. Evidently, the onset is triggered by the oscillation of cavity resonance. At the upper cavity surface around the overhung tongue, the turbulent boundary layer is disrupted and dominated by the large scale turbulence (vortices).

With a laminar uniform 'plug' flow at the inflow boundary, due to the no-slip wall boundary condition, a boundary layer is naturally formed, developed, and turned turbulent near the overhung tongue of the cavity. No specified boundary layer thickness is given at the inlet. Table 1 demonstrates a comparison of the ex-

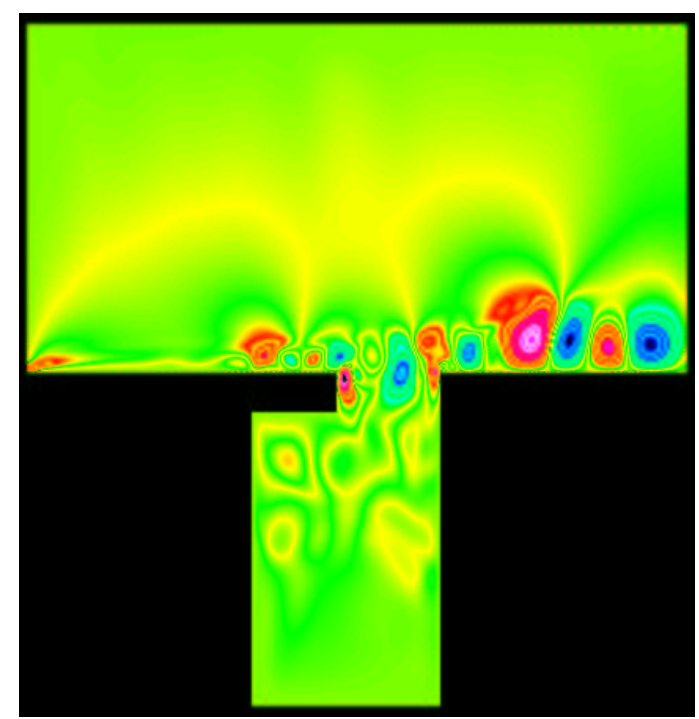

Figure 5: Instantaneous v- velocity contours at time step 740,000 .

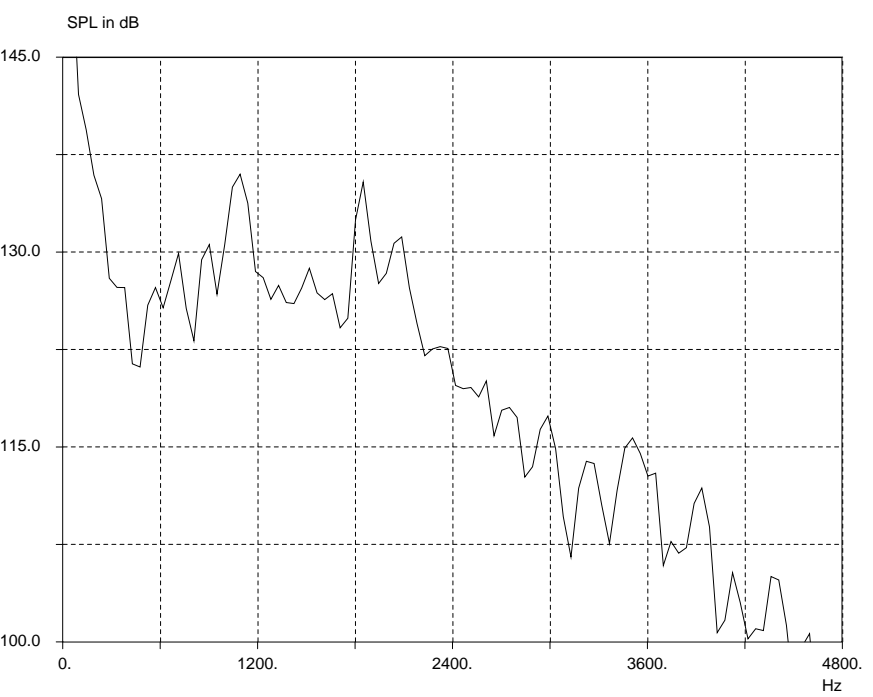

Figure 6: Sound pressure level (SPL) vs. frequency at a low speed of $50 \mathrm{~m} / \mathrm{s}$. 
Table 1: Comparison of experimental and computed tone noises at $50 \mathrm{~m} / \mathrm{s}$

\begin{tabular}{|c|c|c|c|c|c|c|}
\hline $\begin{array}{c}\text { Exp. Freq. } \\
\mathrm{Hz}\end{array}$ & $\begin{array}{c}\text { Exp. SPL } \\
\mathrm{dB}\end{array}$ & $\begin{array}{c}\text { Comput. Freq. } \\
\mathrm{Hz}\end{array}$ & $\begin{array}{c}\text { Comput. SPL } \\
\mathrm{dB}\end{array}$ & $\begin{array}{c}\text { Compt. Freq. } \\
\text { Hz } \\
\text { lrg. domain }\end{array}$ & $\begin{array}{c}\text { Comput. SPL } \\
\text { dB } \\
\text { lrg. domain }\end{array}$ & Noise origin \\
? - unknown
\end{tabular}

perimental and computed data.

From Table 1, it is seen that for the fluid-dynamic feedback tone, the numerical results agree very well with the experimental data, $1837 \mathrm{~Hz}$. vs. $1824 \mathrm{~Hz}$ and $135 \mathrm{~dB}$ vs. $134 \mathrm{~dB}$. in SPL, with a binwidth (or bandwidth) of $47 \mathrm{~Hz}$. The error in frequency or SPL is less than 1\%, although Henderson stated that experimentally, "when multiple tones occur in the spectrum, the preferred or dominant mode often changes randomly. This can result in a change of $3 \mathrm{~dB}$ or more in the peak sound pressure level.' [15]. For the Hemholtz cavity resonance, the frequencies are $2062 \mathrm{~Hz}$ vs. $2012 \mathrm{~Hz}$ with $2 \%$ error, while the error in SPL is larger possibly due to the reason just mentioned above. In addition, as shown in Table 1, multiple tone peaks with lower SPL described by Henderson [15] as tones of unknown origins are also simulated.

Some of these tone peaks of unknown origins in Fig. 6 and Table 1 at the low frequency end seem to be subharmonics, e.g. $1075 \mathrm{~Hz}$ vs. $2062 \mathrm{~Hz}$. Subharmonics are often generated through vortex pairing, the excessively strong subharmonic spikes could be due to the 2-D simulation of a 3-D flow. Such spikes are absent when a coarse grid is used (Fig. 7).

\subsection{Consistency tests}

The same cavity noise problem is also computed with different grid size and larger computational domain dimensions to ensure consistent and reasonable numerical results are obtained. As mentioned above, we are trying to simulate the large scale turbulence itself. The more refined the grid, the more details of the turbulence emerge, as is experienced with a lot of LES (large eddy simulation) computations. In contrast to a deterministic flow computation problem, grid independence may not be tested in a rigorous way for the present noise problem. However, to a certain extent in a statistical way, some general agreements on frequencies may still be available for different grids. Several other consistency tests such as running with a larger computational domain and longer time, as well as imposing a specified boundary layer thickness are also conducted in the following sub-
$\mathrm{SPL}$ in $\mathrm{dB}$, coarse grid

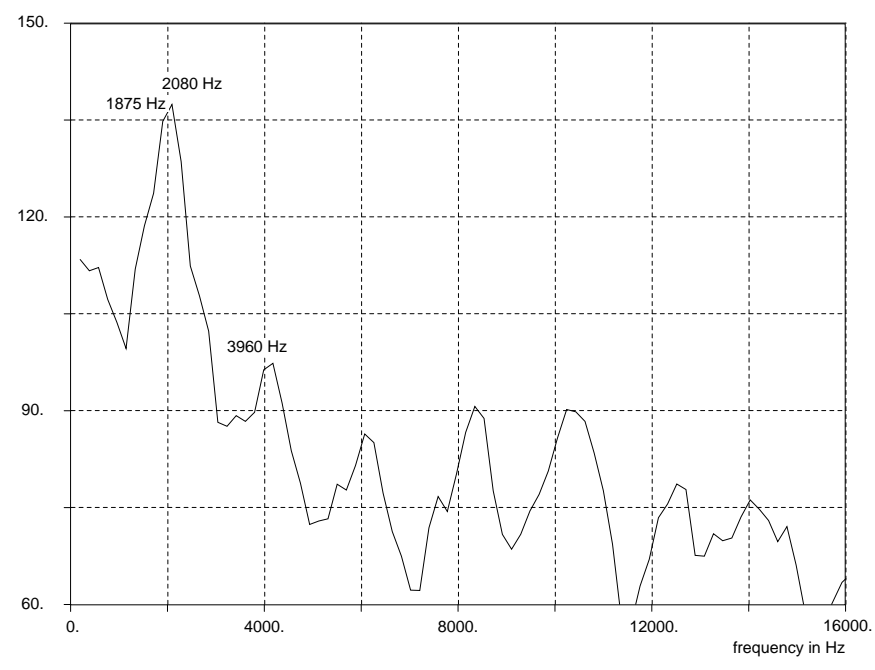

Figure 7: Sound pressure level (SPL) vs. frequency at flow speed of $50 \mathrm{~m} / \mathrm{s}$ with a coarse grid.

sections. When comparing with the case of flow speed $50 \mathrm{~m} / \mathrm{s}$ in $\S 4.1$, it is referred to as the 'standard case'.

4.2.1 Test of parallel computations The cavity noise problems are large scale computational problems in the sense that a huge number of explicit time marching steps are needed. In order to reduce the turnaround time of the computation, parallel computation is adopted. The unstructured triangulated grid is first partitioned into subdomains by METIS [16], then MPI [17] is used to carry out data exchange between different CPU processors in the PC cluster. Sketch of the parallel computation can be found in $[12,13]$.

Here, the same 'standard' cavity noise problem at flow speed of $50 \mathrm{~m} / \mathrm{s}$ is tested on PC clusters with different number of processors ( 8 and 14) to confirm that the parallel computations yield identical numerical results.

4.2.2 Test with a coarse grid This cavity noise problem at flow speed $50 \mathrm{~m} / \mathrm{s}$ was calculated with a coarser grid for grid independence test. Similarly, 


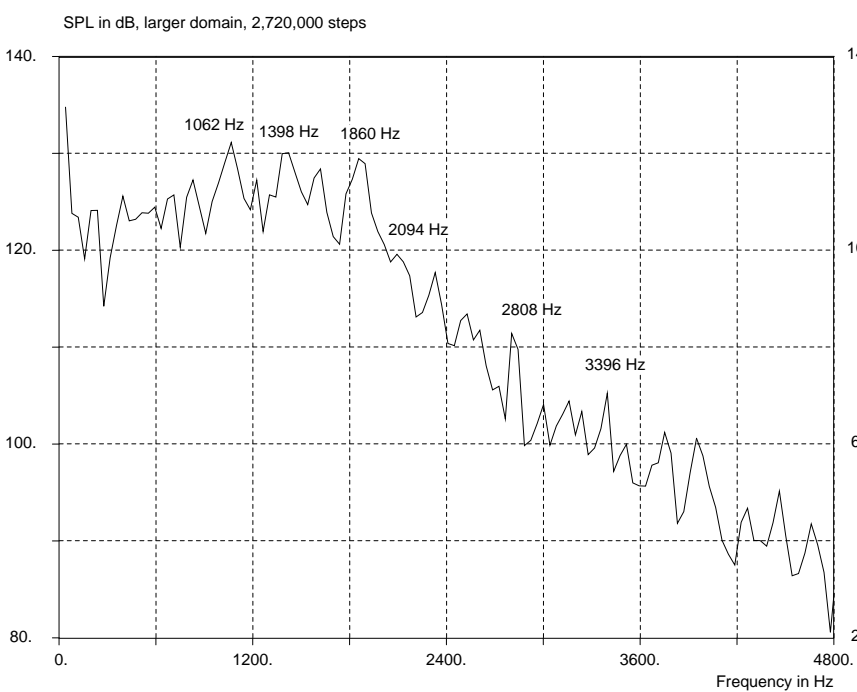

Figure 8: Sound pressure level (SPL) vs. frequency at 50 $\mathrm{m} / \mathrm{s}$ with larger domain and 2,720,000 marching steps.

nonuniform rectangular grid is used before it is triangulated. Although in the refined grid area $\Delta x=0.00625$ is about the same, $\Delta y=.0114$ is almost twice as large in the cavity opening area comparing to the current grid in use. Still, good agreement for the major modes with experimental data and the current simulation was obtained. As shown in Fig. 7, the two shoulder points of the highest spike correspond to $1875 \mathrm{~Hz}, 134.8 \mathrm{~dB}$ and $2080 \mathrm{~Hz}$, $137 \mathrm{~dB}$. Since the binwidth (or bandwidth) is about 190 $\mathrm{Hz}$ in this case, it is not clear that if they represent the fluid dynamic feed back oscillation or the fluid resonance mode, or both and form a broad peak. However, in terms of frequency, they are in good agreement with the experimental data of $1824 \mathrm{~Hz}$ and $134 \mathrm{~dB}$ (fluid dynamic feedback) and the computed data of $1837 \mathrm{~Hz}$ and $135 \mathrm{~dB}$ with the 'standard' grid (Table 2).

For the resonance mode, it is observed that the dominant frequency $(2080 \mathrm{~Hz})$ matches the experimental data well but the SPLs are higher $(137 \mathrm{~dB})$, the grid independence is thus somehow justified. But the tone peaks of unknown origins (broadband noises) are muffled, persistent higher harmonics are generated.

\subsubsection{Test on a larger computational domain} and longer run The problem is also run with a large domain $(-2.8 \leqslant x \leqslant 4,0 \leqslant y \leqslant 4)$. The grid size in this problem is about the same as in the 'standard case' in the critical area, i.e. the cavity interior and around the opening, but grows faster towards the inlet, outflow and top boundaries. Thus, a larger computational domain is formed. With a time step size of $\Delta t=0.003$, a much longer time of 2.72 million steps is run in attempt to find out the long term behavior of the simulation. The
$\mathrm{SPL}$ in $\mathrm{dB}, 2.2 \mathrm{~cm}$ B.L. thickness,

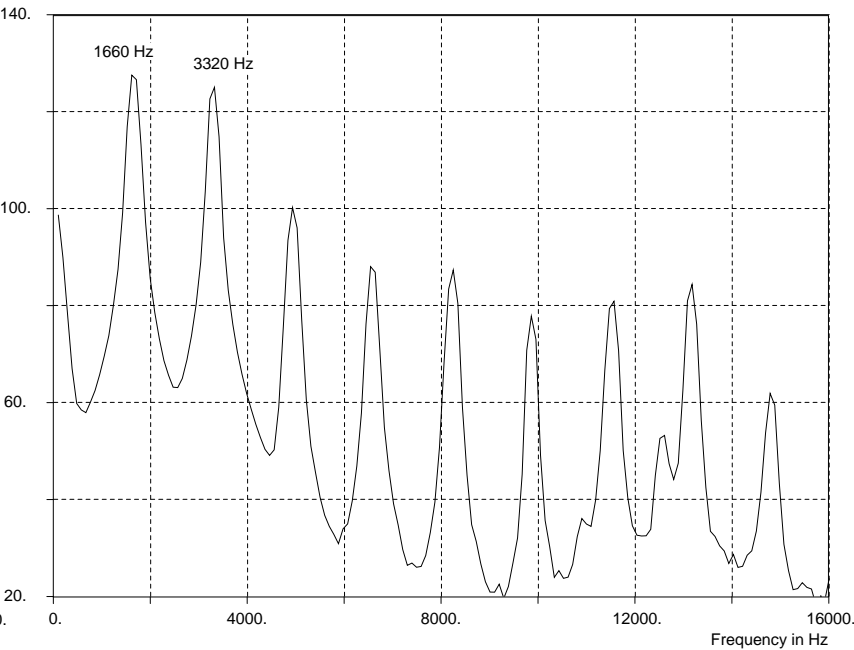

Figure 9: Sound pressure level (SPL) vs. frequency at 50 $\mathrm{m} / \mathrm{s}$, with $2.2 \mathrm{~cm}$ thick boundary layer imposed.

numerical results are presented in Fig. 8. Comparisons to the experimental data and the 'standard case' are still demonstrated in Table 1. Generally speaking, for the dominant oscillation modes, the aeroacoustic feedback and flow resonance, the error is about $2 \%$ which is quite acceptable, despite their lower SPLs as expected due to some coarse grid size. The performance is quite similar to the 'standard case' in that the subharmonic-like high SPL tones appear at the low frequency portion of the spectrum, possibly due to the 2-D simulation for 3-D flow.

It is now demonstrated that, at least for the present simulation, the size of the computational domain (i.e., locations of the boundaries) and the long time running have little influence on the major numerical results.

4.2.4 Tests with artificially imposed boundary layer thickness In all the above numerical simulations, a simple uniform plug flow is imposed at the inlet boundary. In this subsection, prescribed u-velocity profiles are imposed at the inlet boundary to mimic the actual turbulent boundary layers. Given a boundary thickness $\delta$, the formal $\mathrm{u}$-velocity profile at the inlet boundary is specified as:

$$
u=U_{0}(y / \delta)^{1 / 7} .
$$

In the following two test cases, the same computational domain and grid as in the 'standard case' are employed. In the first case, a thick turbulent boundary layer of 2.2 $\mathrm{cm}$ is imposed at the inlet. After 1.4 million time steps, the spectrum at the assigned point on the cavity vertical wall is depicted in Fig. 9. The prominent peaks correspond to $1660 \mathrm{~Hz}, 127 \mathrm{~dB}$ and $3320 \mathrm{~Hz}, 125 \mathrm{~dB}$. Evidently, the second peak is the first harmonic of the first 
Table 2: comparison of experimental and computed tone noises at $50 \mathrm{~m} / \mathrm{s}$, with coarse grid

\begin{tabular}{|c|c|c|c|c|c|c|}
\hline $\begin{array}{c}\text { Exp. Freq. } \\
\mathrm{Hz}\end{array}$ & $\begin{array}{c}\text { Exp. SPL } \\
\mathrm{dB}\end{array}$ & $\begin{array}{c}\text { Comput. Freq. } \\
\mathrm{Hz}\end{array}$ & $\begin{array}{c}\text { Comput. SPL } \\
\mathrm{dB}\end{array}$ & $\begin{array}{c}\text { Compt. Freq. } \\
\mathrm{Hz} \\
\text { coarse grid }\end{array}$ & $\begin{array}{c}\text { Comput. SPL } \\
\mathrm{dB} \\
\text { coarse grid }\end{array}$ & Noise origin \\
\hline 930 & 103 & 1075 & 135 & - & - & $?$ \\
\hline 1340 & 107 & 1326 & 127 & - & - & $?$ \\
\hline 1824 & 134 & 1837 & 135 & 1875 & 134.8 & feedback \\
\hline 2016 & 113 & 2062 & 130 & 2080 & 137 & resonance \\
\hline 2848 & 106 & 2962 & 116 & - & - & $?$ \\
\hline 3552 & 111 & 3513 & 115 & 3960 & 98 & $?$ \\
\hline
\end{tabular}

$\mathrm{SPL}$ in $\mathrm{dB}, 1.2 \mathrm{~cm}$ B.L. thickness

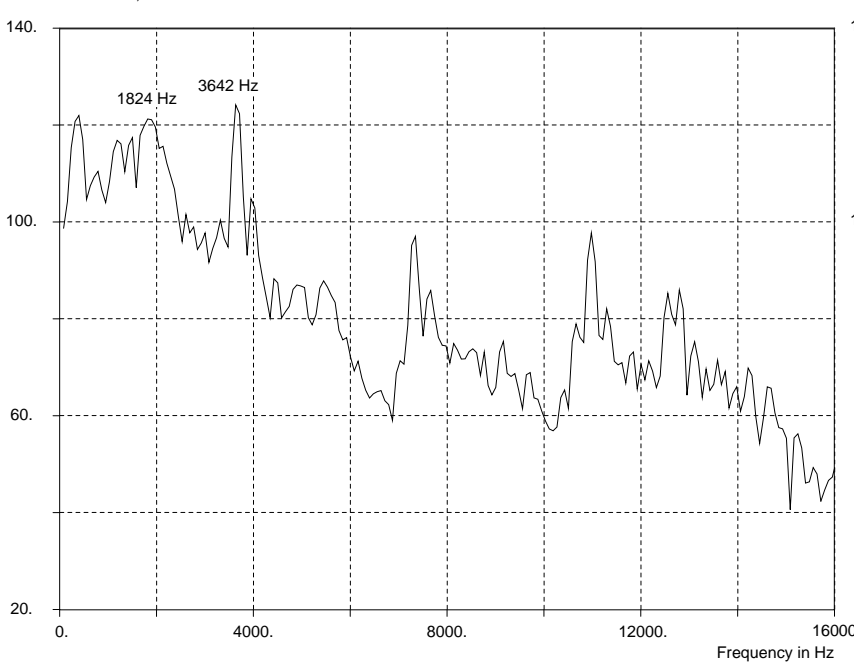

Figure 10: Sound pressure level (SPL) vs. frequency at $50 \mathrm{~m} / \mathrm{s}$, with $1.2 \mathrm{~cm}$ thick artificial boundary layer at inlet

peak. Note that the spectrum exhibits ample high frequency harmonics of a single tone, this implies numerically, there exists a highly nonlinear wave. The pattern is quite different from the experimental one [15].

In the second case, the imposed boundary layer thickness at the inlet is reduced to $1.2 \mathrm{~cm}$. Figure 10 illustrates the spectrum after 1.4 million time steps. A slightly larger $\Delta t=0.003$ is used. This time, the prominent peaks correspond to $1824 \mathrm{~Hz}, 121 \mathrm{~dB}$ and $3642 \mathrm{~Hz}, 124$ $\mathrm{dB}$. It is also noted that in Fig. 10, to the left of the peak of $1824 \mathrm{~Hz}$, there is a smaller peak at about $2090 \mathrm{~Hz}$, which may correspond to the flow resonance frequency of $2016 \mathrm{~Hz}$. In terms of frequency, the results are exceedingly good, almost agreeing exactly with the experimental data for 'thin' boundary layer [15]. There is no surprise since $\delta=1.2 \mathrm{~cm}$ is exactly the boundary layer thickness from the experiment. However, in terms of SPL, the numerical ones are still low probably because the grid is still not fine enough. In addition, high SPL

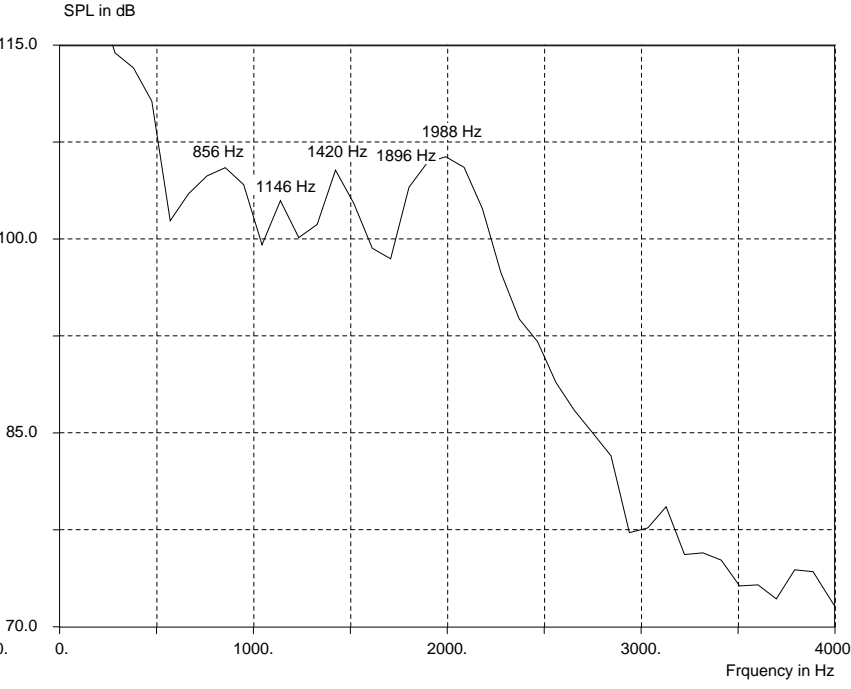

Figure 11: Sound pressure level (SPL) vs. frequency at a low speed of $26.8 \mathrm{~m} / \mathrm{s}$.

unwanted peaks appear at the low frequency end of the spectrum possibly due to 2-D simulation.

The numerical experiments show that the prescribed boundary layer thickness has considerable effect on the spectrum and SPL. Without any knowledge or hint about the boundary layer, an arbitrarily prescribed boundary layer thickness may not work well and it is probably best to let the flow develop its own.

\subsection{Noise at at a lower flow speed of $26.8 \mathrm{~m} / \mathrm{s}$}

Numerical experiments were also conducted for an even lower flow speed of $26.8 \mathrm{~m} / \mathrm{s}$ for the same cavity geometry. Table 3 lists a comparison of the experimental and computed data. As in the 'standard case', uniform plug flow is imposed at the inlet boundary.

As in the previous case, from Table 3, it is observed that for the fluid-dynamic feedback tone, the numerical results agree very well with the experimental data, 1146 Hz. vs. $1168 \mathrm{~Hz}$ and $103 \mathrm{~dB}$ vs. $99 \mathrm{~dB}$ in SPL, with a binwidth (or bandwidth) of $95 \mathrm{~Hz}$. The error in fre- 
Table 3: Comparison of experimental and computed tone noises at $26.8 \mathrm{~m} / \mathrm{s}$

\begin{tabular}{|c|c|c|c|c|}
\hline $\begin{array}{c}\text { Exp. Freq. } \\
\mathrm{Hz}\end{array}$ & $\begin{array}{c}\text { Exp. SPL } \\
\mathrm{dB}\end{array}$ & $\begin{array}{c}\text { Comput. Freq. } \\
\mathrm{Hz}\end{array}$ & $\begin{array}{c}\text { Comput. SPL } \\
\mathrm{dB}\end{array}$ & Noise origin \\
\hline 928 & 97 & 856 & 105 & $?$ - unknown \\
\hline 1168 & 99 & 1146 & 103 & feedback \\
\hline 1380 & 95 & 1420 & 105 & $?$ \\
\hline 1890 & 103 & 1896 & 105 & $?$ \\
\hline 1984 & 101 & 1988 & 106 & resonance \\
\hline
\end{tabular}

quency or SPL is about $3-4 \%$. For the cavity resonance, the frequencies are $1988 \mathrm{~Hz}$ vs. $1984 \mathrm{~Hz}$ with $0.2 \%$ error, and the SPLs are respectively $106 \mathrm{~dB}$ and $101 \mathrm{~dB}$. There are also other noise tones of unknown origin, the errors in frequencies and SPLs with these tones are larger. Similar to the case at flow speed of $50 \mathrm{~m} / \mathrm{s}$, their SPL are relatively high, possibly due to the 2-D simulation of a 3-D flow. From Fig. 11, there is probably another tone of unknown origin - the one at $1896 \mathrm{~Hz}$. Its frequency is so close to the resonance frequency $1988 \mathrm{~Hz}$ and the binwidth is $95 \mathrm{~Hz}$, making it hard to distinguish the two frequencies. The two frequency spikes coalesce into a single broad peak. According to Henderson's observation from the experiments [15], well defined discrete frequencies do not appear consistently until the flow velocity reaches $35 \mathrm{~m} / \mathrm{s}$. Based on this argument, the numerical results for the lower flow speed are indeed in very good agreement with the experiments.

4.3.1 Test with a coarse grid The same problem was tested on a coarse grid [18] similar to the one in §4.2.2. Due to lack of resolution, the SPL is much lower and only one spike at $1916 \mathrm{~Hz}$ appears in the spectrum, which may correspond to either the resonance frequency at $1984 \mathrm{~Hz}$ with $3.5 \%$ error or the frequency spike of unknown origin at $1890 \mathrm{~Hz}$. In terms of frequency, the approximation is acceptable.

\section{Concluding Remarks}

In this paper, a MILES type numerical approach is adopted to simulate the cavity tone noises arising from large scale turbulence. No explicit turbulence or subgrid models or acoustic models are used. As there are two dominant oscillation modes - aeroacoustic feedback and flow resonance competing with each other, accompanied by some tones of unknown origins, or broadband noise, it is important to select carefully the grid size and other parameters in the computations. Generally good results are obtained and compare favorably to the experimental ones. although 2-D simulation for 3-D flows may generate some excessively strong subharmonics towards the lower end of the spectrum.
In the MILES type approach, we attempt to simulate only the large scale turbulence, which is believed to be the tone noise generating source. The smaller scale turbulences (higher frequencies and wave numbers) are practically ignored without an explicit subgrid scale model, since we are interested primarily in the aeroacoustic data. The CE/SE method is a scheme for solving conservation laws. When the divergence theorem (2) is applied to find the cell average, it also plays a role as a filter ( such as the one used in LES approach). High frequency and high wave number disturbances are filtered out and aliasing errors are unlikely to occur. As pointed out by Fureby [6], choosing the parameters or limiters in the MILES approach, is somewhat equivalent to choosing a SGS model in the conventional LES approach. The choice is not straightforward in the presence of complicated flows.

\section{References}

[1] J.E.Rossiter, "Wind Tunnel Experiments of the Flow over rectangular Cavities at Subsonic and transonic Speeds", Aero.Res. Counc. R \& M., 3438, 1964.

[2] M.S.Howe, "Edge, cavity and aperture tones at very low Mach numbers". J. Fluid Mech., vol.330, pp. 61-84, 1997.

[3] L.Chatellier, J.Laumonier, and Y.Gervais, "Aeroacoustics Coupling in Cavities at Low Mach Number", AIAA Paper 2001-2131.

[4] J.P.Boris, F.F.Grinstein,E.S.Oran, and R.J.Kolbe, "New Insights into Large Eddy Simulation", Fluid Dynamics Research, vol.10, No.4-6, pp.199-227, 1992.

[5] E.Garnier, M.Mossi, P.Sagaut, P.Comte, and M.Deville, "On the Use of Shock-Capturing Schemes for Large Eddy Simulation", J. Comput. Phys., vol.153, pp.273-311, 1999. 
[6] C.Fureby and F.F.Grinstein, "Monotonically Integrated Large Eddy Simulation of Shear Flow", AIAA J. , vol.37, No.5, pp.544-556, 1999.

[7] Chang, S.-C., Wang, X.-Y. and Chow, C.-Y., "The Space-Time Conservation Element and Solution Element Method-A New High Resolution and Genuinely Multidimensional Paradigm for Solving Conservation Laws," J. Comp. Phys. vol. 159, pp. 89-136 (1999).

[8] Wang, X.-Y. and Chang S.-C., “ A 2-D Nonsplitting Unstructured Triangular Mesh Euler Solver Based on the Space-Time Conservation Element and Solution Element Method" C.F.D. J. vol. 8, pp309-325 (1999).

[9] Loh, C. Y., Hultgren, L. S. and Chang S.-C., "Computing Waves in Compressible Flow Using the Space-Time Conservation Element Solution Element Method," AIAA J., Vol. 39, pp. 794-801 (2001).

[10] Loh, C. Y. and Zaman, K.B.M.Q., “ Numerical Investigation of Transonic Resonance with a Convergent-Divergent Nozzle", AIAA J., Vol. 40, No. 12, pp. 2393-2401 (2002).

[11] Loh, C. Y., Hultgren, L. S., and Jorgenson, P. C. E., 'Near Field Screech Noise Computation for an Underexpanded Supersonic Jet by the CE/SE Method', AIAA Paper 2001-2252, (2001).
[12] Loh, C. Y., Himansu, A. Wang, X.-Y. and Jorgenson, P. C. E., "Computation of an Underexpanded 3-D Rectangular jet by the CE/SE Method", AIAA Paper 2001-0986 (2001).

[13] Loh, C. Y., Himansu, A., and Hultgren, L.S., "A 3D Navier-Stokes Solver with Unstructured Hexahedral grid for Computations of Near Field jet Screech Noise" AIAA Paper 2003-3207 (2003).

[14] Loh, C. Y., "On a Nonreflecting Boundary Condition for Hyperbolic Conservation Laws" AIAA Paper 2003-3975 (2003).

[15] B. Henderson, "Category 6, Automobile Noise Involving Feedback-Sound generation by Low Speed Cavity Flows", in "Third Computational Aeroacoustics (CAA) Workshop on Benchmark problems", NASA CP-2000-209790, August, 2000.

[16] Karypis, G. and Kumar, V. "Multilevel k-way Partitioning Scheme for Irregular Graphs", Univ. of Minnesota Dept. of Comp. Sc./Army HPC Research Center Tech. Report 95-064 (1995).

[17] mpi website: http://www-unix.mcs.anl.gov/mpi

[18] Loh, C. Y., Chang, Sin-C., Wang, X.-Y. and Jorgenson, P. C. E., "Gap Noise Computation by the CE/SE Method" ASME paper, FEDSM-200118136. 


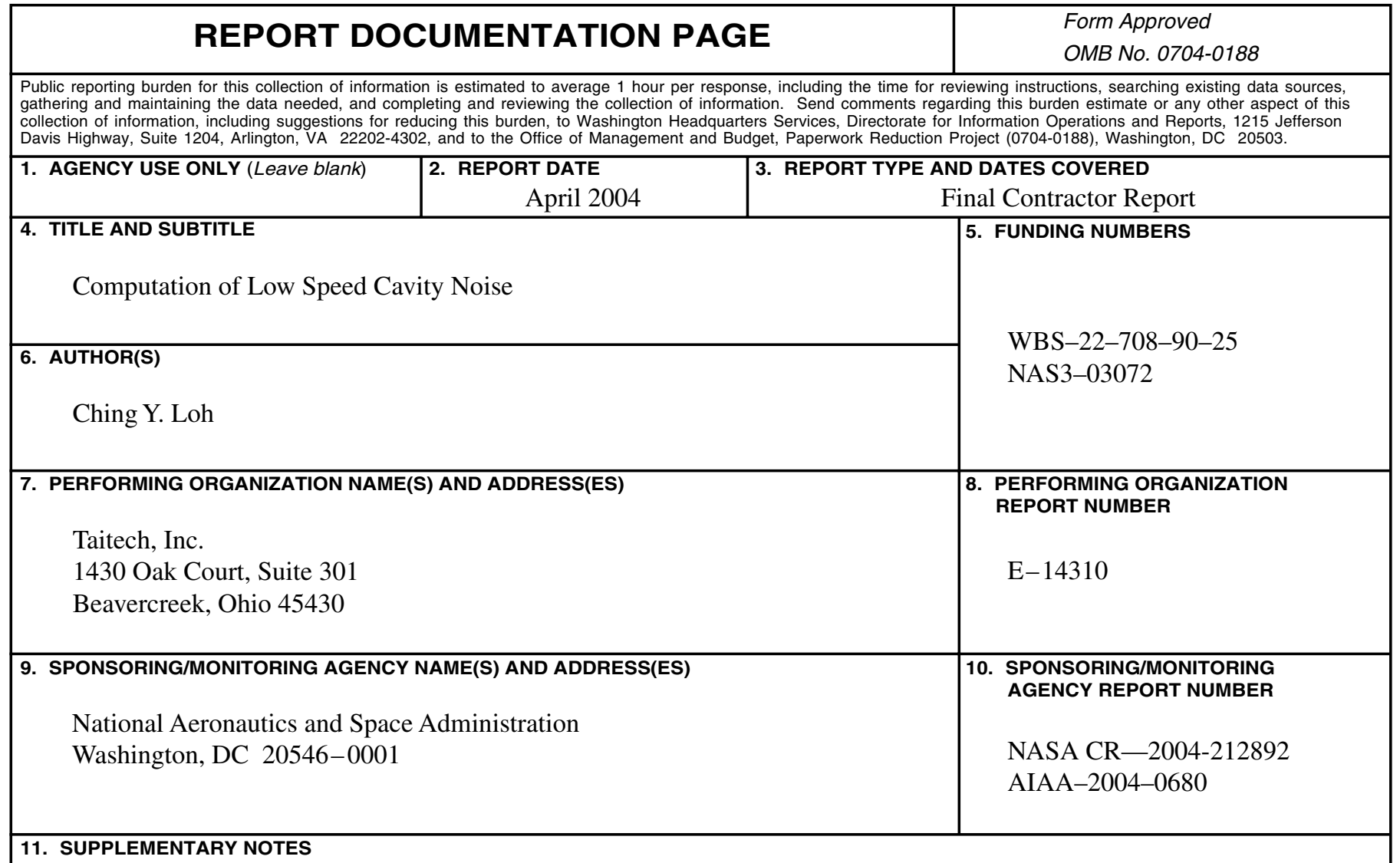

Project Manager, Richard A. Blech, Turbomachinery and Propulsion Systems Division, NASA Glenn Research Center, organization code 5880, 216-433-3657.

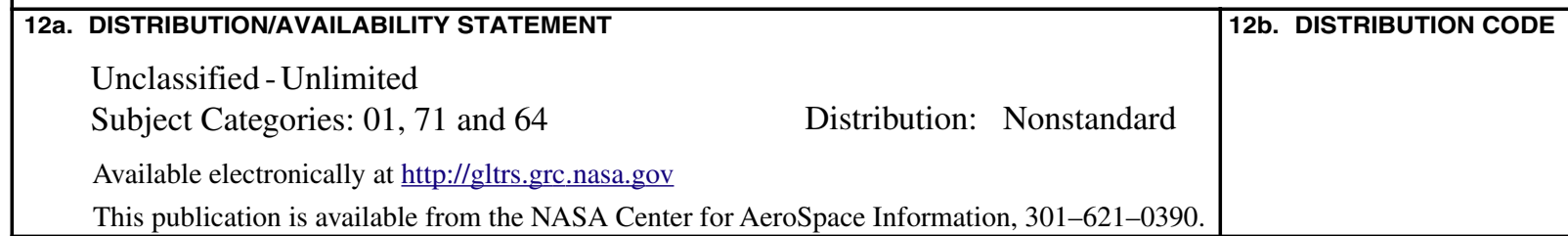

13. ABSTRACT (Maximum 200 words)

Cavity noise is an important noise source. It is generated primarily by wall-jet shear layer and vortex shedding and an aeroacoustic feedback loop. At low flow speed, the situation is further complicated by the presence of the Helmholtz resonance of the cavity. In this paper, the space-time conservation element and solution element (CE/SE) method is employed to numerically study the near-field noise of a cavity at low flow speeds. The numerical approach is of the MILES type (monotonically integrated large eddy simulation). The computed frequencies and sound pressure levels (SPL) compare well with the experimental findings.

\begin{tabular}{|c|c|c|c|}
\hline \multicolumn{3}{|c|}{$\begin{array}{l}\text { 14. SUBJECT TERMS } \\
\text { Cavity noise; CE/SE method }\end{array}$} & $\begin{array}{l}\text { 15. NUMBER OF PAGES } \\
16 \\
\text { 16. PRICE CODE } \\
\text { 20. LIMITATION OF ABSTRACT }\end{array}$ \\
\hline
\end{tabular}



